\title{
Comparative diagnostic and prognostic performances of the hematoxylin-eosin and phospho-histone H3 mitotic count and Ki-67 index in adrenocortical carcinoma
}

\author{
Eleonora Duregon ${ }^{1}$, Luca Molinaro ${ }^{2}$, Marco Volante ${ }^{1}$, Laura Ventura ${ }^{3}$, Luisella Righi ${ }^{1}$, \\ Stefania Bolla ${ }^{2}$, Massimo Terzolo ${ }^{4}$, Anna Sapino ${ }^{2}$ and Mauro G Papotti ${ }^{1}$ \\ ${ }^{1}$ Department of Oncology, University of Turin at San Luigi Hospital, Orbassano, Italy; ${ }^{2}$ Department of \\ Medical Sciences, University of Turin, Turin, Italy; ${ }^{3}$ Department of Statistics, University of Padua, \\ Padova, Italy and ${ }^{4}$ Department of Clinical and Biological Sciences, University of Turin at San Luigi Hospital, \\ Orbassano, Italy
}

\begin{abstract}
Mitotic count on hematoxylin and eosin slides is a fundamental morphological criterion in the diagnosis and grading of adrenocortical carcinoma in any scoring system employed. Moreover, it is the unique term strongly associated with patient's prognosis. Phospho-histone H3 is a mitosis-specific antibody, which was already proven to facilitate mitotic count in melanoma and other tumors. Therefore, a study was designed to assess the diagnostic and prognostic role of phospho-histone $\mathrm{H} 3$ in $\mathbf{5 2}$ adrenocortical carcinomas, comparing manual and computerized count to standard manual hematoxylin- and eosin-based method and Ki-67 index. Manual hematoxylin and eosin and phospho-histone $\mathrm{H} 3$ mitotic counts were highly correlated $(r=0.9077, P<0.0001)$, better than computer-assisted phospho-histone H3 evaluations, and had an excellent inter-observer reproducibility at Bland-Altman analysis. Three of $\mathbf{1 5}$ cases having $<\mathbf{5}$ mitotic figures per $\mathbf{5 0}$ high-power fields by standard count on hematoxylin and eosin gained the mitotic figure point of Weiss Score after a manual count on phospho-histone $\mathrm{H} 3$ slides. Traditional mitotic count confirmed to be a strong predictor of overall survival $(P=0.0043)$, better than phospho-histone H3-based evaluation $(P=0.051)$, but not as strong as the Ki-67 index $(P<0.0001)$. The latter further segregated adrenocortical carcinomas into three prognostic groups, stratifying cases by low ( $<20 \%)$, intermediate $(20-50 \%)$, and high $(>50 \%) \mathrm{Ki}-67$ values. We conclude that (a) phosphohistone $\mathrm{H} 3$ staining is a useful diagnostic complementary tool to standard hematoxylin and eosin mitotic count, enabling optimal mitotic figure evaluation (including atypical mitotic figures) even in adrenocortical carcinomas with a low mitotic index and with a very high reproducibility; (b) Ki-67 proved to be the best prognostic indicator of overall survival, being superior to the mitotic index, irrespective of the method (standard on hematoxylin and eosin or phospho-histone H3-based) used to count mitotic figures.

Modern Pathology (2014) 27, 1246-1254; doi:10.1038/modpathol.2013.230; published online 17 January 2014
\end{abstract}

Keywords: mitotic index; phospho-histone H3; adrenocortical carcinoma; Ki-67; diagnosis; prognosis

Mitotic count on hematoxylin- and eosin-stained slides still is a crucial morphological parameter in the diagnostic work up and grading of a wide range of neoplasms of several organs (skin melanocytic lesions, sarcomas, breast cancers, lung and gastroentero-pancreatic neuroendocrine tumors, and

Correspondence: Professor M Volante, MD, Department of Oncology, University of Turin at San Luigi Hospital, regione Gonzole 10, 10043 Orbassano, Turin, Italy.

E-mail: marco.volante@unito.it

Received 13 August 2013; accepted 23 October 2013; published online 17 January 2014 endocrine neoplasms and brain tumors). Accordingly, mitotic figure assessment is a fundamental criterion in the diagnosis of adrenocortical carcinoma, whichever scoring system (the Weiss ${ }^{1}$ or the van Slooten $^{2}$ ones) or algorithmic approach (Reticulin Algorithm $^{3,4}$ or the stepwise discriminant diagnostic system $^{5}$ ) is employed. This is even more true in those adrenocortical carcinomas with few or borderline features of malignancy, in which the accurate identification of a single parameter can be crucial to reach the threshold of malignancy (for example, in the Weiss Score for classical adrenocortical carcinoma or the Lin-Weiss-Bisceglia 
scheme for oncocytic tumors. ${ }^{6}$ ) Moreover, among all parameters included in the Weiss Score, mitotic rate is the unique criterion strongly associated with patient prognosis. For this reason, a mitotic countbased grading of adrenocortical carcinoma has been also proposed recently, with a cutoff of 20 mitotic figures per 50/high-power fields to separate low from high-grade adrenocortical carcinomas. ${ }^{7}$

In front of such critical role of mitotic figures, technical and inter-observer variability influence the precision of mitotic count in a considerable way. Mitotic figures can be obscured by crushing and staining artifacts, or mimicked by apoptosis and karyorrhexis, especially in necrotic areas. In addition, the mitotic index should be necessarily counted in 50 high-power fields in order to improve accuracy in low-proliferating tumors. Unfortunately, mitotic index evaluation is time consuming and it is heavily influenced by the experience and meticulousness of the pathologist performing the count. ${ }^{8}$ In a recent study of Weiss Score reproducibility in adrenocortical carcinoma diagnosis, the parameter 'mitotic count $\geq 5 / 50$ high-power fields' was found to have a 'moderate' inter-observer reproducibility that was significantly improved (up to a 'substantial' agreement) after a specific training of the involved observers. ${ }^{9}$ However, the interobserver agreement of actual mitotic count was not assessed.

Mitotic figure identification could be facilitated by the use of mitosis-specific stainings. The commercially available antibody to phospho-histone H3 (also known as Ser-10) ${ }^{10}$ targets the phosphorylated form of the core histone protein $\mathrm{H} 3$ in position 10 of a serine amino acid (another antibody is specific for phosphorylation in serine 28). This protein is detected maximally during mitotic chromosome condensation in early prophase and negligibly at any other times (including apoptosis). ${ }^{11,12}$ Phospho-histone H3 immunostaining has been shown to be a reliable and easy method for mitotic index evaluation in melanoma, with both diagnostic ${ }^{13-18}$ and prognostic $^{17,19-21}$ implications. Moreover, it has been also successfully applied in different neoplasms of the breast, ${ }^{22-26}$ meninges, ${ }^{27-29}$ brain, ${ }^{30}$ lung, ${ }^{31}$ soft tissues, ${ }^{32-34}$ esophagus, ${ }^{35}$ female genital tract, ${ }^{36,37}$ prostate, ${ }^{38}$ and urothelium, ${ }^{39}$ as well as in cytology material from pancreatic endocrine tumors ${ }^{40}$ and urothelial carcinoma. ${ }^{41}$

The Ki-67 index represents an alternative method for assessing the proliferative potential in adrenocortical tumors and has already been shown to differentiate adrenocortical adenoma from carcinoma, ${ }^{42-49}$ and to predict tumor behavior. ${ }^{42,50,51}$ However, the use of the Ki-67 index is not officially incorporated in the diagnostic work up of adrenocortical carcinoma, which still largely relies on histomorphological parameters. ${ }^{52}$

On the basis of the foregoing, our study was designed on a relatively large series of 52 adrenocortical carcinomas to assess the diagnostic and
Table 1 Clinical pathological features of 52 adrenocortical carcinomas analyzed for phospho-histone H3 expression

\begin{tabular}{ll} 
Parameter & \\
\hline F/M ratio & 1.26 \\
Age, mean (years) (range) & $47(20-79)$ \\
Functional status (not known: & Not functioning: 20 \\
& Functioning: 28 \\
Mean size (cm) (range) & $11.2(2-30)$ \\
Mean weight (g) (range) & $422(8-3100)$ \\
Adrenocortical carcinoma & Classical: 35 \\
variant & Myxoid: 9 \\
& Oncocytic (pure/mixed): 8 (5/3) \\
Weiss Score distribution & $3-4: 12$ \\
& $5-6: 15$ \\
& $7-8-9: 25$ \\
ENSAT stage (not known: 5) & $1-2: 31$ \\
& $3-4: 16$ \\
Disease status (lost to FU: 2) & NED/DOC: 14 \\
& AWD: 6 \\
& DOD: 30 \\
Median overall survival (months) 53 (1-180)
\end{tabular}

Abbreviations: AWD, alive with disease; DOC, died of other cause; DOD, died of disease; F, female; FU, follow-up; M, male; NED, no evidence of disease.

prognostic roles of phospho-histone $\mathrm{H} 3$ immunostaining in comparison with both manual and computer-assisted hematoxylin and eosin mitotic count and Ki-67 index determination.

Our results show that (a) phospho-histone H3 staining may greatly facilitate mitotic figure evaluation even in cases with a low mitotic index, as it is highly correlated to hematoxylin and eosin mitotic index evaluation, has a similar reproducibility, but is easier to assess; (b) in our series Ki-67 is superior to mitotic index (either calculated on hematoxylin and eosin- or phospho-histone H3-stained slides) in predicting patients' prognosis.

\section{Materials and methods}

\section{Tissue Collection}

Fifty-two adrenocortical tumors having a Weiss Score $\geq 3^{1}$ (including nine myxoid and eight oncocytic) were retrieved from the pathology files of the University of Turin at San Luigi Hospital. Malignancy in the eight oncocytic tumors was also confirmed by using the more accurate Bisceglia scheme. ${ }^{6}$ The majority of these patients were treated at our Institution, which serves as a referral center for adrenocortical carcinoma in Italy. The clinical and pathological features of all cases belonging to this data set (summarized in Table 1) have already been reported ${ }^{3,53-55}$ and were updated. The study received ethical approval from the local Review Board of our Institution.

\section{Immunohistochemistry}

All available hematoxylin- and eosin-stained slides were reviewed, and a representative paraffin block 
was selected for each case. Five-micrometer-thick serial paraffin sections were processed by means of immunohistochemistry using antibodies against phospho-histone H3 (Cell Marque-Roche, rabbit polyclonal antibody, prediluted) and Ki-67 (Dako, clone MIB-1, diluted 1/150). A biotin-free, dextran chain-based detection system (EnVysion, Dako) and diaminobenzidine as the chromogen, or alkaline phosphatase and a red detection kit (Ventana Medical Systems, Tucson, AZ, USA) were used according to standard protocols for Ki-67 and phospho-histone H3, respectively.

\section{Morphological and Phospho-histone H3-Based Mitotic Index Assessment and ki-67 Evaluation}

Two observers (ED and LM) independently assessed the mitotic rate and Ki-67 proliferation index on the same hematoxylin and eosin, phospho-histone H3 and Ki-67 slide sets. Mitotic figures were counted in 50 consecutive high-power fields both on hematoxylin and eosin- and phospho-histone H3-stained sections. In phospho-histone H3-stained slides, mitotic figures were recognized by the presence of a positive staining and consistent morphologic features. The Ki-67 index was determined by counting 1000 cells in hot spots and it was reported as the percentage of positive nuclei.

In addition, all sections stained for phosphohistone $\mathrm{H} 3$ and Ki-67 were scanned at $\times 20$ magnification using the Aperio XT system (Nikon Instruments Europe, Aperio Technologies, Vista, CA, USA). Svs (ScanScope Virtual Slides) extension files were created and images were then visualized with the Aperio Image Scope software. Regions of interest (ROIs) were selected within the whole tumor area by an expert pathologist (LM). Necrotic areas, residual adrenal gland, and connective tissue were excluded. The slide analysis was performed using an FDAapproved algorithm 'Aperio Nuclear V9' (by Aperio Technologies) for nuclear immunohistochemical signal detection. To obtain the number of fields for each slide, the entire area digitally scanned was divided for a $\times 40$ objective area (with $0.5 \mathrm{~mm}$ field diameter). The total number of positive nuclei was then divided for the number of fields and the mitotic index was obtained.

\section{Statistical Analysis}

GraphPad Prism version 5.0 and free-software R (http://www.r-project.org/) programs were used for statistical analyses. The level of significance was set at $P<0.05$. Pearson's coefficients and BlandAltman plots ${ }^{56}$ were used to analyze correlation and agreement between pairs of measurements on hematoxylin and eosin, phospho-histone H3 (manual and PC-assisted) and $\mathrm{Ki}-67$, respectively. To analyze the impact on survival, a Kaplan-Meier estimate of survival distribution was performed using

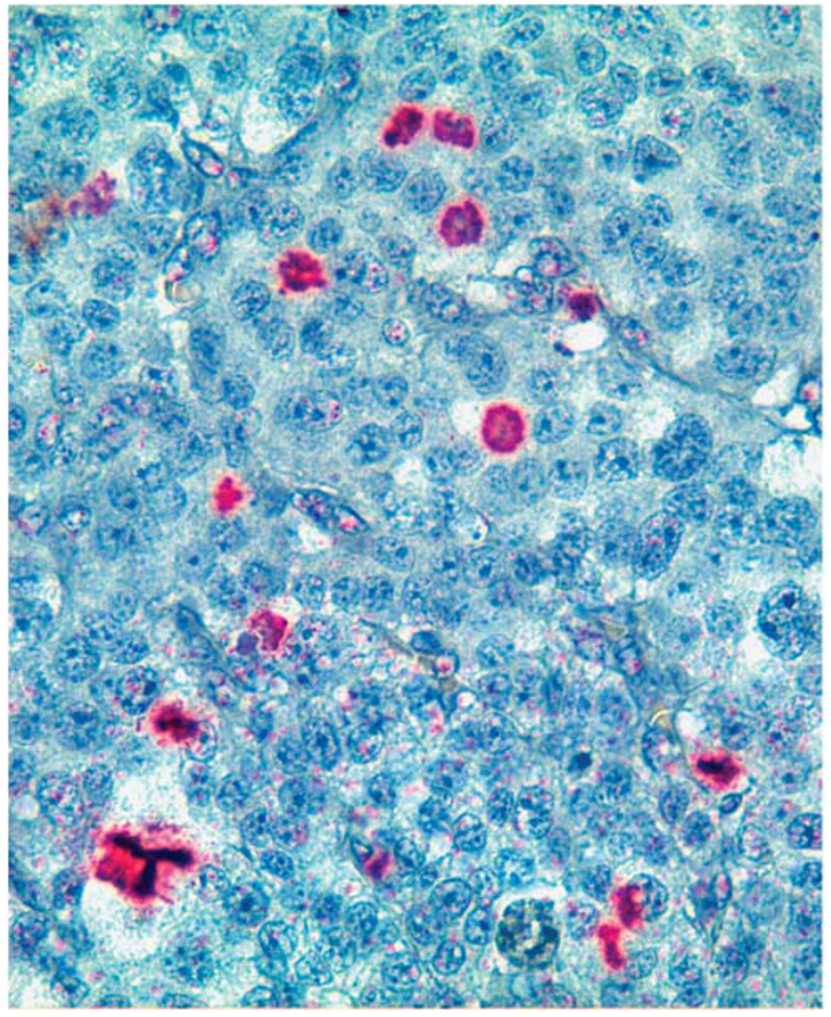

Figure 1 Representative example of phospho-histone H3 mitotic figure immunostaining of adrenocortical carcinoma (original magnification $\times 400$ ).

median and quartile values of each parameter. Overall survival curves were compared using the log-rank test.

\section{Results}

\section{Diagnostic Role of Phospho-Histone H3}

Phospho-histone H3 mitotic count: correlation with hematoxylin and eosin mitotic rate. The mitotic index manually counted on hematoxylin and eosinstained slides had a mean value of 23 mitotic figures per 50 high-power fields (range 0-115). Forty-eight cases $(92 \%)$ were adequate for phospho-histone H3 evaluation (Figure 1). Both typical and atypical mitotic figures could be easily identified and rapidly counted. The mean manual mitotic rate in phosphohistone H3-stained slides was 28 mitotic figures per 50 high-power fields (range 0-140), whereas using the computer-assisted method it was 3.279 positive nuclei per high-power field (range 0-12). After a square root transformation, hematoxylin and eosin and phospho-histone $\mathrm{H} 3$ manual and digitalized mitotic counts were normally distributed. Manual hematoxylin and eosin and phospho-histone H3 mitotic rates were highly correlated $(r=0.9077$, $P<0.0001)$, with a mean difference of $-2.39(95 \%$ confidence interval: $0.35-3.10$ at Bland-Altman plot analysis, Figure 2a). Computer-assisted phospho-histone H3 mitotic rate correlated better with 
a

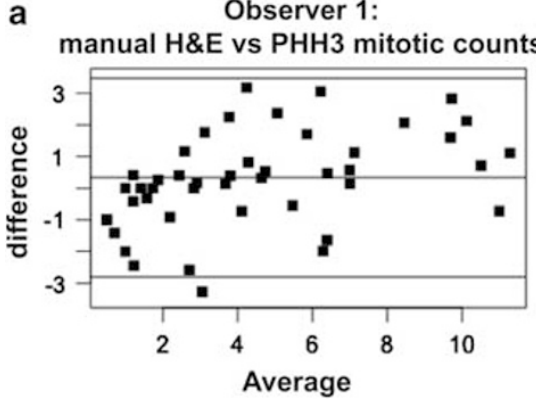

b Observer 2:

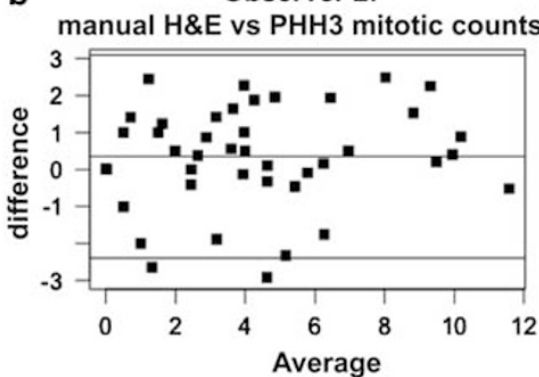

Figure 2 Correlation between hematoxylin and eosin (H\&E) and phospho-histone H3 (PHH3) mitotic count in two separate observers. Bland-Altman plots demonstrating the mean differences between manual hematoxylin and eosin and phospho-histone H3 mitotic count for observer $1(-2.39(95 \% \mathrm{CI}=0.35-3.10))(\mathbf{a})$ and observer $2(-2.80(95 \% \mathrm{CI}=0.35-3.10))(\mathbf{b})$.

manual phospho-histone H3 $(r=0.7365, P<0.0001)$ than with manual hematoxylin and eosin $(r=0.6125, P<0.0001)$ mitotic counts.

Focusing on the subgroup of 15 cases that did not reach five mitotic figures per 50 high-power fields according to standard hematoxylin and eosin-based mitotic count, three of them gained the mitotic figure item of the Weiss Score when the count was manually made on phospho-histone H3-stained slides. Interestingly, seven of these 15 low-proliferating tumors belonged to the oncocytic variant, although the cases with the highest phospho-histone H3 mitotic index in this group were adrenocortical carcinomas of the classical type. Seven cases with an hematoxylin and eosin-based mitotic count between five and eight mitotic figures per 50 high-power fields moved from a mean value of seven mitotic figures per 50 high-power fields with hematoxylin and eosin to 13 with phospho-histone H3.

Reproducibility of mitotic count: phospho-histone H3 compared with hematoxylin and eosin method. The independent evaluation of the same set by a second observer produced the mean values of mitotic index similar to the first observer (25-range 0-128-and 33-range 0-140-for hematoxylin and eosin- and phospho-histone H3-based mitotic counts, respectively). As for the first observer, hematoxylin and eosin and phospho-histone H3 mitotic rates showed a high correlation $(r=0.8931$, $P<0.0001)$, with a mean difference of $-2.80(95 \%$ confidence interval: $0.35-3.10$ at Bland-Altman plot, Figure 2b). Manually determined mitotic rates on hematoxylin and eosin- and phospho-histone H3-stained slides showed a very high inter-observer correlation $(r=0.9671, P<0.0001$ and $r=0.9698$, $P<0.0001$, respectively). The high degree of interobserver agreement of hematoxylin and eosin and phospho-histone H3 mitotic counts was also demonstrated at Bland-Altman plot analysis (hematoxylin and eosin mean difference: $-1.97,95 \%$ confidence interval: - 0.45-1.08; phospho-histone H3 mean difference: -2.17 , 95\% confidence interval: - 0.499-1.174; Figures 3a and b).
Comparison between phospho-histone H3 mitotic count and Ki-67 proliferation index. The mean values of Ki-67 proliferation index (evaluable in all but one case) were $30 \%$ (range 1-85\%) and $20 \%$ (range 1-71\%) in manual and computer-assisted counts, respectively. After the same square root transformation, manual Ki-67 proliferation index correlated with manual hematoxylin and eosin $(r=0.7195, \quad P<0.0001)$ and phospho-histone H3 $(r=0.7886, P<0.0001)$ mitotic counts. In both cases, the correlation was lower than what observed between phospho-histone $\mathrm{H} 3$ and hematoxylin and eosin. The inter-observer reproducibility of manual Ki-67 proliferation index was good $(r=0.8180, P<0.0001)$ but lower than what observed for both hematoxylin and eosin and phospho-histone H3 mitotic figure counts, with a mean difference between the two evaluators of -2.7622 (95\% confidence interval: 0.5419-3.8499 at Bland-Altman plot, Figure 3c). Computer-assisted and manual Ki-67 determinations were also reciprocally correlated $(r=0.8070, \quad P<0.0001$ and $r=0.8890$, $P<0.0001$ for the two observers, respectively).

\section{Prognostic Role of Mitotic Count and Ki-67 Proliferation Index}

Survival analysis. The median follow-up of our patient population was 40 months (range, 1-199 months). Mitotic index evaluation on hematoxylin and eosin slides, also in this limited case series, confirmed to be a strong predictor of overall survival, using the median cutoff value for this study population (11 mitoses in 50 high-power fields). Phospho-histone H3-based mitotic evaluation (using the median value of 9 as the cutoff point) did not perform better than hematoxylin and eosin mitotic index assessment, and it was indeed associated to overall survival with borderline statistical significance, only (Figure 4). A further survival analysis, based on quartiles for these two markers, was not able to significantly substratify patient groups. The Ki-67 proliferation index using the median value $(22 \%$ in this study population) showed the highest significance in terms of overall 
survival. A further stratification was obtained by dividing Ki-67 values into low $(<20 \%)$, intermediate $(20-50 \%)$, and high ( $>50 \%)$, and proved highly significant to segregate three prognostic groups in our series (Figure 5).

\section{Discussion}

In this study, we found that phospho-histone H3 immunostaining is an alternative, faster, and reliable method to highlight mitotic figures, a fundamental diagnostic parameter for adrenocortical carcinoma, even in cases with low mitotic activity. However, immunohistochemically assisted mitotic index evaluation using the anti-phospho-histone $\mathrm{H} 3$ antibody does not improve the prognostic role of mitotic count, whereas the Ki-67 proliferation index was the best indicator of patient's prognosis.

\section{Phospho-Histone H3 Role to Highlight Mitotic Figures} as a Diagnostic Tool

Accuracy of mitotic index evaluation may be influenced by different factors, including technical artifacts due to DNA crushing or pyknosis on the one side, and the misinterpretation of apoptotic bodies or dying cells (especially in areas of extensive cellular necrosis) for mitotic figures on the other. Therefore, apart from being a time-consuming exercise, assessment of mitotic figures in 50 high-power fields is often poorly reproducible among observers. Phospho-histone H3 immunostaining has been demonstrated to specifically detect mitotic figures in several human tumors ${ }^{19,22,27,30-32}$ but has never been tested in adrenocortical carcinomas, so far. This is surprising, as it is well known that the recognition of this tumor type largely relies on mitotic index evaluation, as one of the nine factors included in the Weiss Score ${ }^{1}$ or in other diagnostic algorithms..$^{3-5}$ Therefore, a first aim of this study was to assess the usefulness of phosphohistone $\mathrm{H} 3$ immunohistochemical staining (evaluated either manually or with an automated PC-based approach) as a surrogate of morphological mitotic index evaluation. Our data confirm and expand to adrenocortical carcinoma the reported high concordance between morphologically and phospho-histone H3-assessed mitotic counts, with a high statistical significance for both manual and computerassisted $^{19,22-26,40}$ phospho-histone H3 counting. Phospho-histone H3 immunostaining allowed a rapid count of mitotic figures and is also useful for the recognition of atypical mitotic figures, a second relevant parameter included in the Weiss Score (Figure 1). The 'atypical mitotic figure' parameter is relevant also in diagnostic schemes specifically designed for pure oncocytic tumors, ${ }^{6}$ an adrenocortical tumor variant often associated to a very low proliferative potential, which could therefore take the maximal advantage from phospho-histone H3 immunostaining.
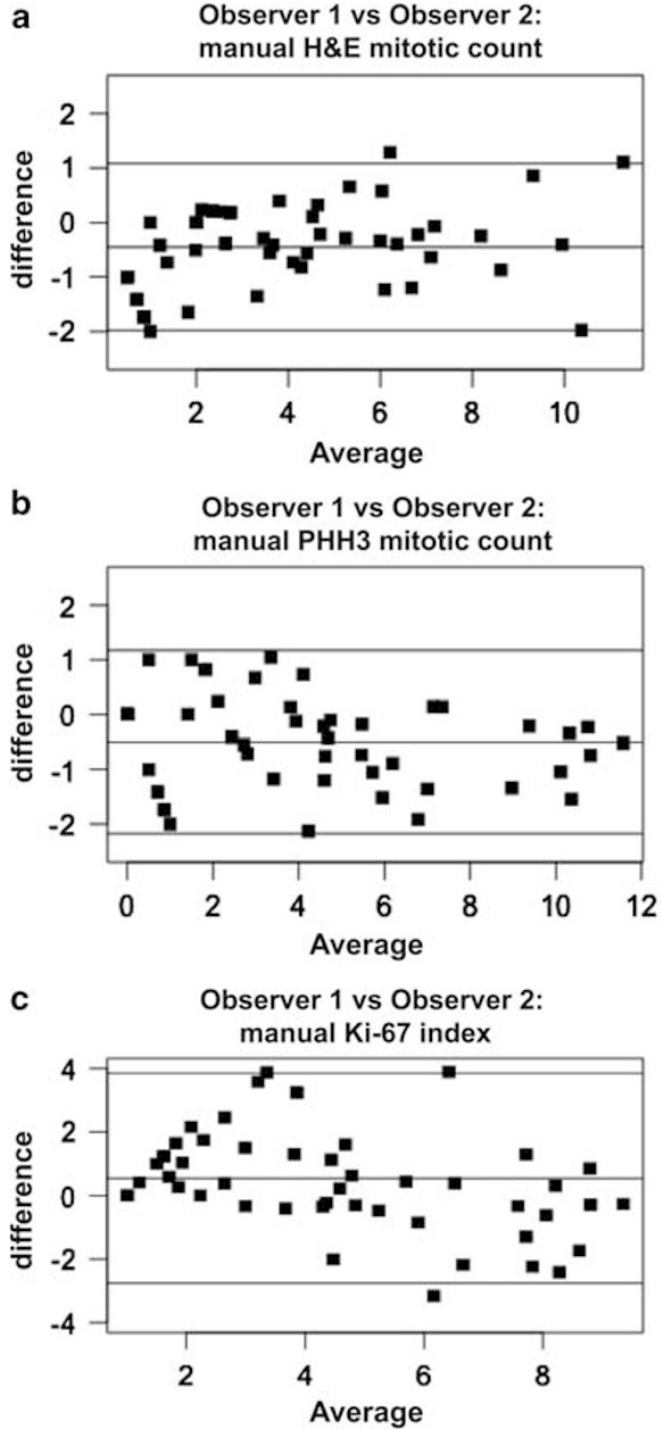

Figure 3 Inter-observer agreement. Bland-Altman plots demonstrating the mean difference between mitotic counts produced by two observers based on hematoxylin and eosin (H\&E) (a) $(-1.9769, \quad 95 \% \mathrm{CI}=-0.4486-1.0797)$, phospho-histone H3 (PHH3)-stained slides (b) $(-2.1725,95 \% \mathrm{CI}=-0.4992-1.174)$, and the Ki-67 proliferation index (c) $(-2.7622,95 \% \mathrm{CI}=0.5419-$ 3.8499).

Inter-Observer Reproducibility of Hematoxylin and Eosin Mitotic Count, Phospho-Histone H3 and Ki-67

We analyzed the inter-observer reproducibility of these three methods. Both hematoxylin and eosinand phospho-histone H3-based mitotic counts proved to have an excellent inter-observer agreement, higher than the Ki-67 proliferation index. ${ }^{14,17}$

Therefore, it seems that irrespective of the diagnostic system employed, a phospho-histone H3 immunohistochemical staining can represent an useful tool to simplify mitotic figure screening and to improve the diagnostic accuracy in adrenocortical tumors. 


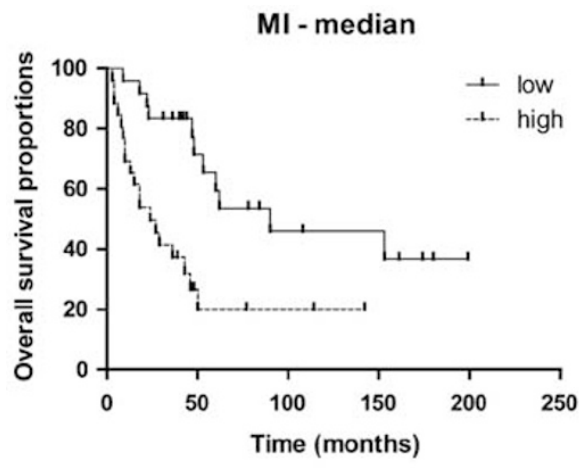

LogRank (Mantel Cox): $p=0.0016$

HR: 0.29 [0.13-0.63]

Median survival:

1-2: 90; 3-4: 24 months

PHH3 - median

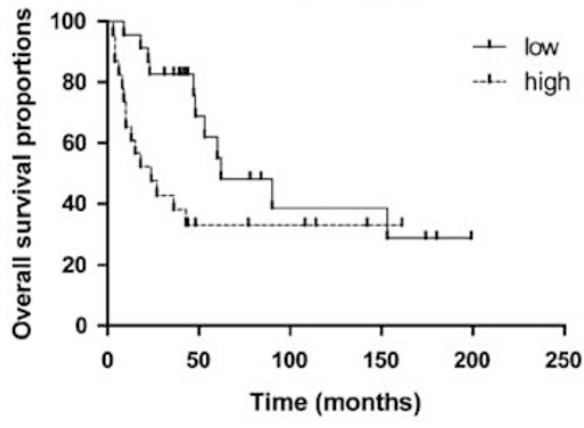

LogRank (Mantel Cox): $p=0.051$

HR: 0.45 [0.20-1.00]

Median survival:

1-2: 62; 3-4: 24 months

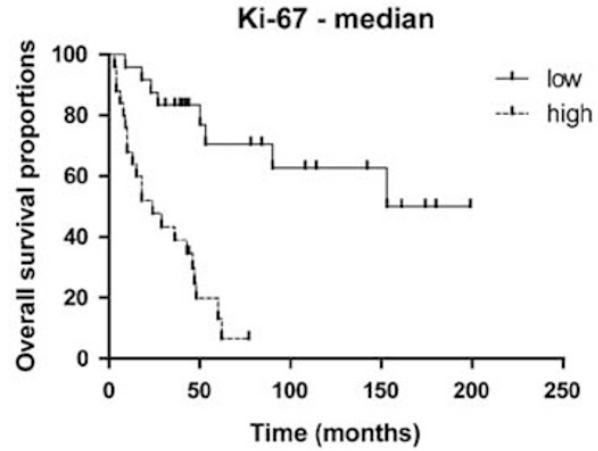

LogRank (Mantel Cox): $p<0.0001$

HR: 0.16 [0.07-0.36]

Median survival:

1-2: undefined; 3-4: 24 months

Figure 4 Overall survival analysis. Overall survival curves for mitotic index based on hematoxylin and eosin (H\&E) and phospho-histone H3 (PHH3) and the Ki-67 proliferation index, segregated according to the median values.

Ki-67 - prognostic groups

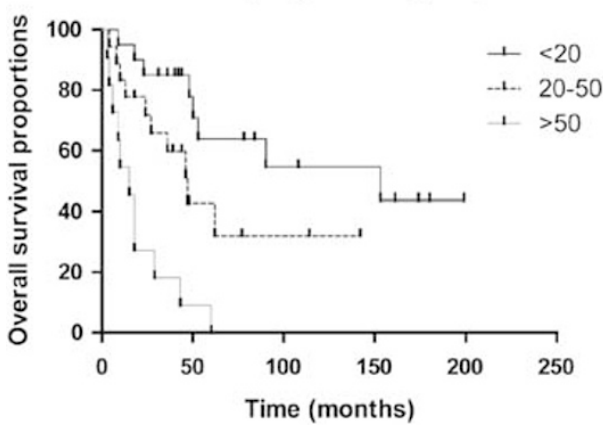

LogRank (Mantel Cox): $p<0.0001$

$<20$ vs 20-50: $\mathrm{p}=0.072$;

20-50 vs $>50: p=0.027$;

Median survival:

$<20 \%: 153$ months;

20\%-50\%: 47 months;

>50\%: 15 months

Figure 5 Prognostic stratification according to the Ki-67 index. Overall survival curves of adrenocortical carcinomas segregated into three prognostic groups according to the Ki-67 proliferation index. 
Phospho-Histone H3 Manual Count in LowProliferating Adrenocortical Carcinomas

Mitotic count in highly proliferating tumors is generally not a problem, at least for the purpose of determining whether the mitotic number exceeds the required threshold of 5/50 high-power fields in the Weiss Score or in the reticulin algorithm. Therefore, a major interest was devoted to adrenocortical carcinoma cases having a low mitotic activity, which may represent a diagnostic challenge, especially in the case of simultaneous limited presence of other malignancy-related parameters. Moreover, some adrenocortical carcinoma variants, with special reference to oncocytic adrenocortical tumors, are characterized by the intrinsic occurrence of some Weiss parameters that may produce an overestimation of malignancy when the Weiss Score is applied. The accurate determination of the remaining parameters, including the mitotic index and atypical mitotic figures, is therefore even more crucial to support a diagnosis of carcinoma.

In the critical subgroup of cases lacking the mitotic figure item (according to the Weiss Score), phospho-histone H3 highlighted more mitotic figures only in cases of the classical type. It could therefore be suggested that in front of a lowproliferating tumor, phospho-histone H3 immunostaining might be a helpful complement to standard mitotic figure determination in hematoxylin and eosin slides. By contrast, computer-assisted phospho-histone $\mathrm{H} 3$ evaluation failed to maintain a high concordance rate in cases with low mitotic activity. The reasons for this observation are not clear but possibly reflect the different criteria for selecting areas of interest adopted by individual operators or by the automated system. However, based on this latter finding, it seems reasonable to conclude that computer-assisted phospho-histone $\mathrm{H} 3$ evaluation is not associated to a better diagnostic performance, as compared with standard mitotic index evaluation and it is not recommended in the field of adrenocortical carcinoma diagnosis.

\section{Prognostic Role of Phospho-Histone H3}

Although not specifically designed for identifying a new prognostic factor in adrenocortical carcinoma, this study allowed also a comparison of phosphohistone $\mathrm{H} 3$ immunostaining with morphologically defined mitotic index, which is the most relevant pathological prognostic factor in adrenocortical carcinomas. Indeed, phospho-histone H3 did not improve the prognostic value reported for standard mitotic count on hematoxylin and eosin slides, and its performance was even slightly worse, based on a pure statistical ground. In this context, our data are in agreement with those of Ladstein ${ }^{16}$ on thick cutaneous melanoma, although in many tumorsincluding melanoma itself-the prognostic value of phospho-histone H3-based mitotic count was found to be stronger than conventional hematoxylin and eosin-based counts. ${ }^{20,25,37}$

\section{Impact of Ki-67 Evaluation as Compared with Mitotic Count in Adrenocortical Carcinoma}

Although not extensively investigated in adrenocortical carcinoma, it is well known in other tumor types that mitotic index evaluated on hematoxylin and eosin and Ki-67 proliferation index are reciprocally correlated. ${ }^{8,57}$ Therefore, we additionally compared phospho-histone H3 data with Ki-67 values and found a very high correlation, with special reference to phospho-histone H3 manual counting. The impact of Ki-67 determination at the diagnostic level is however not well established in adrenocortical carcinoma, and from a practical point of view the value of this observation is limited. Conversely, a major interest was related to the use of Ki-67 as an alternative or complementary tool to the mitotic index in adrenocortical carcinoma prognostication. Our data, representative of the largest series of adrenocortical carcinomas analyzed so far in this setting, clearly show that Ki-67 is superior to mitotic count (irrespective of the method of estimation) in terms of prognostic stratification of adrenocortical carcinoma patients, at least concerning overall survival. This allowed to propose three prognostic subgroups based on different Ki-67 cutoffs $(<20,20$ 50 and $>50 \%$ ) with the highest statistical performance. This latter observation, although with all the limitations due to the lack of adequate information for multivariate analysis (including subanalysis of treated versus untreated patients), is strong enough to implement the concept of the need of a grading system in adrenocortical carcinomas, as recently proposed, ${ }^{58}$ and expands the potential tools available to define patients' risk stratification.

\section{Conclusions}

Phospho-histone H3 immunostaining is a valid, accurate, and reproducible surrogate marker of standard mitotic count in adrenocortical tumors, helping to optimize the time-consuming traditional mitotic figure search in 50 high-power fields. In particular, phospho-histone $\mathrm{H} 3$ is recommended for low-proliferating tumors to avoid underestimation of the 'mitotic figure parameter' of Weiss and other diagnostic systems. Conversely, it is not superior as a prognostic parameter; in this setting, Ki-67 proliferation index determination proved to be the most powerful tool to predict patient's survival.

\section{Acknowledgments}

This work was supported by grants from the Italian Association for Cancer Research (AIRC, Milan, grant no. IG/10795/2010 to MP) and the University of Turin (ex-60\% grants to MV and MP). 


\section{Disclosure/conflict of interest}

The authors declare no conflict of interest.

\section{References}

1 Weiss LM, Medeiros LJ, Vickery AL Jr. Pathologic features of prognostic significance in adrenocortical carcinoma. Am J Surg Pathol 1989;13:202-206.

2 van Slooten H, Schaberg A, Smeenk D, et al. Morphologic characteristics of benign and malignant adrenocortical tumors. Cancer 1985;55:766-773.

3 Volante M, Bollito E, Sperone P, et al. Clinicopathological study of a series of 92 adrenocortical carcinomas: from a proposal of simplified diagnostic algorithm to prognostic stratification. Histopathology 2009;55:535-543.

4 Duregon E, Fassina A, Volante $\mathrm{M}$, et al. The reticulin algorithm for adrenocortical tumor diagnosis: a multicentric validation study on 245 unpublished cases. Am J Surg Pathol 2013;37:1433-1440.

5 Blanes A, Diaz-Cano SJ. Histologic criteria for adrenocortical proliferative lesions: value of mitotic figure variability. Am J Clin Pathol 2007;127:398-408.

6 Bisceglia M, Ludovico O, Di Mattia A, et al. Adrenocortical oncocytic tumors: report of 10 cases and review of the literature. Int J Surg Pathol 2004;12: 231-243.

7 Miller BS, Gauger PG, Hammer GD, et al. Proposal for modification of the ENSAT staging system for adrenocortical carcinoma using tumor grade. Langenbecks Arch Surg 2010;395:955-961.

8 Molenaar WM, Plaat BE, Berends ER, et al. Observer reliability in assessment of mitotic activity and MIB-1determined proliferation rate in pediatric sarcomas. Ann Diagn Pathol 2000;4:228-235.

9 Tissier F, Aubert S, Leteurtre E, et al. Adrenocortical tumors: improving the practice of the Weiss system through virtual microscopy: a National Program of the French Network INCa-COMETE. Am J Surg Pathol 2012;36:1194-1201.

10 Hendzel MJ, Wei Y, Mancini MA, et al. Mitosisspecific phosphorylation of histone H3 initiates primarily within pericentromeric heterochromatin during G2 and spreads in an ordered fashion coincident with mitotic chromosome condensation. Chromosoma 1997;106:348-360.

11 Juan G, Traganos F, James WM, et al. Histone H3 phosphorylation and expression of cyclins A and B1 measured in individual cells during their progression through G2 and mitosis. Cytometry 1998;32:71-77.

12 Hendzel MJ, Nishioka WK, Raymond Y, et al. Chromatin condensation is not associated with apoptosis. J Biol Chem 1998;273:24470-24478.

13 Ikenberg K, Pfaltz M, Rakozy C, et al. Immunohistochemical dual staining as an adjunct in assessment of mitotic activity in melanoma. J Cutan Pathol 2012;39: 324-330.

14 Schimming TT, Grabellus F, Roner M, et al. pHH3 immunostaining improves interobserver agreement of mitotic index in thin melanomas. Am J Dermatopathol 2012;34:266-269.

15 Casper DJ, Ross KI, Messina JL, et al. Useof anti-phosphohistone H3 immunohistochemistry to determine mitotic rate in thin melanoma. Am J Dermatopathol 2010;32:650-654.
16 Ladstein RG, Bachmann IM, Straume O, et al. Ki-67 expression is superior to mitotic count and novel proliferation markers PHH3, MCM4 and mitosin as a prognostic factor in thick cutaneous melanoma. BMC Cancer 2010;10:140.

17 Angi M, Damato B, Kalirai $\mathrm{H}$, et al. Immunohistochemical assessment of mitotic count in uveal melanoma. Acta Ophthalmol 2011;89:e155-e160.

18 Nasr MR, El-Zammar O. Comparison of pHH3, Ki-67, and survivin immunoreactivity in benign and malignant melanocytic lesions. Am J Dermatopathol 2008; 30:117-122.

19 Hale CS, Qian M, Ma MW, et al. Mitotic rate in melanoma: prognostic value of immunostaining and computer-assisted image analysis. Am J Surg Pathol 2013;37:882-889.

20 Tetzlaff MT, Curry JL, Ivan D, et al. Immunodetection of phosphohistone $\mathrm{H} 3$ as a surrogate of mitotic figure count and clinical outcome in cutaneous melanoma. Mod Pathol 2013;26:1153-1160.

21 Ladstein RG, Bachmann IM, Straume O, et al. Prognostic importance of the mitotic marker phosphohistone H3 in cutaneous nodular melanoma. J Invest Dermatol 2012;132:1247-1252.

22 Gudlaugsson E, Klos J, Skaland I, et al. Prognostic comparison of the proliferation markers mitotic activity index, phosphohistone H3, Ki67, steroid receptors, HER2, high molecular weight cytokeratins and classical prognostic factors in T1-2N0M0 breast cancer. Pol J Pathol 2013;64:1-8.

23 Skaland I, Janssen EA, Gudlaugsson E, et al. The prognostic value of the proliferation marker phosphohistone H3 (PPH3) in luminal, basal-like and triple negative phenotype invasive lymph node-negative breast cancer. Cell Oncol 2009;31:261-271.

24 Skaland I, Janssen EA, Gudlaugsson E, et al. Validating the prognostic value of proliferation measured by Phosphohistone H3 (PPH3) in invasive lymph nodenegative breast cancer patients less than 71 years of age. Breast Cancer Res Treat 2009;114:39-45.

25 Skaland I, Janssen EA, Gudlaugsson E, et al. Phosphohistone H3 expression has much stronger prognostic value than classical prognosticators in invasive lymph node-negative breast cancer patients less than 55 years of age. Mod Pathol 2007;20:1307-1315.

26 Bossard C, Jarry A, Colombeix C, et al. Phosphohistone H3 labelling for histoprognostic grading of breast adenocarcinomas and computer-assisted determination of mitotic index. J Clin Pathol 2006;59:706-710.

27 Fukushima S, Terasaki M, Sakata K, et al. Sensitivity and usefulness of anti-phosphohistone-H3 antibody immunostaining for counting mitotic figures in meningioma cases. Brain Tumor Pathol 2009;26:51-57.

$28 \mathrm{Kim}$ YJ, Ketter R, Steudel WI, et al. Prognostic significance of the mitotic index using the mitosis marker anti-phosphohistone H3 in meningiomas. Am J Clin Pathol 2007;128:118-125.

29 Ribalta T, McCutcheon IE, Aldape KD, et al. The mitosis-specific antibody anti-phosphohistone-H3 (PHH3) facilitates rapid reliable grading of meningiomas according to WHO 2000 criteria. Am J Surg Pathol 2004;28:1532-1536.

30 Colman H, Giannini C, Huang L, et al. Assessment and prognostic significance of mitotic index using the mitosis marker phospho-histone $\mathrm{H3}$ in low and intermediate-grade infiltrating astrocytomas. Am J Surg Pathol 2006;30:657-664. 
31 Tsuta K, Liu DC, Kalhor N, et al. Using the mitosisspecific marker anti-phosphohistone $\mathrm{H} 3$ to assess mitosis in pulmonary neuroendocrine carcinomas. Am J Clin Pathol 2011;136:252-259.

32 Idriss MH, Kazlouskaya V, Malhotra S, et al. Phosphohistone-H3 and Ki-67 immunostaining in cutaneous pilar leiomyoma and leiomyosarcoma (atypical intradermal smooth muscle neoplasm). J Cutan Pathol 2013;40:557-563.

33 Veras E, Malpica A, Deavers MT, et al. Mitosis-specific marker phospho-histone $\mathrm{H} 3$ in the assessment of mitotic index in uterine smooth muscle tumors: a pilot study. Int J Gynecol Pathol 2009;28:316-321.

34 Kapur P, Rakheja D, Balani JP, et al. Phosphorylated histone H3, Ki-67, p21, fatty acid synthase, and cleaved caspase-3 expression in benign and atypical granular cell tumors. Arch Pathol Lab Med 2007;131: 57-64.

35 Nakashima S, Shiozaki A, Ichikawa D, et al. Antiphosphohistone $\mathrm{H} 3$ as an independent prognostic factor in human esophageal squamous cell carcinoma. Anticancer Res 2013;33:461-467.

36 Aune G, Stunes AK, Tingulstad S, et al. The proliferation markers Ki-67/MIB-1, phosphohistone H3, and survivin may contribute in the identification of aggressive ovarian carcinomas. Int J Clin Exp Pathol 2011;4:444-453.

37 Brunner A, Riss P, Heinze G, et al. pHH3 and survivin are co-expressed in high-risk endometrial cancer and are prognostic relevant. Br J Cancer 2012;107:84-90.

38 Braun M, Stomper J, Kirsten R, et al. Landscape of chromosome number changes in prostate cancer progression. World J Urol 2013;31:1489-1495.

39 Gunia S, Kakies C, Erbersdobler A, et al. Scoring the percentage of Ki67 positive nuclei is superior to mitotic count and the mitosis marker phosphohistone H3 (PHH3) in terms of differentiating flat lesions of the bladder mucosa. J Clin Pathol 2012;65:715-720.

40 Draganova-Tacheva $\mathrm{R}$, Bibbo $\mathrm{M}$, Birbe $\mathrm{R}$, et al. The potential value of phosphohistone-h3 mitotic index determined by digital image analysis in the assessment of pancreatic endocrine tumors in fine-needle aspiration cytology specimens. Acta Cytol 2013;57: 291-295.

41 Solomides CC, Birbe RC, Nicolaou N, et al. Does mitosis-specific marker phosphohistone H3 help the grading of upper tract urothelial carcinomas in cell blocks? Acta Cytol 2012;56:285-288.

42 Iino K, Sasano H, Yabuki N, et al. DNA topoisomerase II alpha and Ki-67 in human adrenocortical neoplasms: a possible marker of differentiation between adenomas and carcinomas. Mod Pathol 1997;10:901-907.

43 Nakazumi H, Sasano H, Iino K, et al. Expression of cell cycle inhibitor p27 and Ki-67 in human adrenocortical neoplasms. Mod Pathol 1998;11:1165-1170.

44 Arola J, Salmenkivi K, Liu J, et al. p53 and Ki67 in adrenocortical tumors. Endocr Res 2000;26:861-865.
45 Terzolo M, Boccuzzi A, Bovio S, et al. Immunohistochemical assessment of Ki-67 in the differential diagnosis of adrenocortical tumors. Urology 2001;57: 176-182.

46 Stojadinovic A, Brennan MF, Hoos A, et al. Adrenocortical adenoma and carcinoma: histopathological and molecular comparative analysis. Mod Pathol 2003;16:742-751.

47 Soon PS, Gill AJ, Benn DE, et al. Microarray gene expression and immunohistochemistry analyses of adrenocortical tumors identify IGF2 and Ki-67 as useful in differentiating carcinomas from adenomas. Endocr Relat Cancer 2009;16:573-583.

48 Schmitt A, Saremaslani P, Schmid S, et al. IGFII and MIB1 immunohistochemistry is helpful for the differentiation of benign from malignant adrenocortical tumours. Histopathology 2006;49:298-307.

49 Orhan D, Kale G, Caglar M, et al. Histone mRNA in situ hybridization and Ki 67 immunohistochemistry in pediatric adrenocortical tumors. Virchows Arch 2006; 448:591-596.

50 Morimoto R, Satoh F, Murakami O, et al. Immunohistochemistry of a proliferation marker Ki67/MIB1 in adrenocortical carcinomas: Ki67/MIB1 labeling index is a predictor for recurrence of adrenocortical carcinomas. Endocr J 2008;55:49-55.

51 Vargas MP, Vargas HI, Kleiner DE, et al. Adrenocortical neoplasms: role of prognostic markers MIB-1, P53, and RB. Am J Surg Pathol 1997;21:556-562.

52 Lloyd RV, Tischler AS, Kimura N, et al. Adrenocortical carcinoma. In: De Lellis RA, Lloyd RV, Heitz PU, Eng C (eds). World Health Organization Classification of Tumors of Endocrine Organs: Lyon, France; 2009, pp 137-146.

53 Papotti M, Volante M, Duregon E, et al. Adrenocortical tumors with myxoid features: a distinct morphologic and phenotypical variant exhibiting malignant behavior. Am J Surg Pathol 2010;34:973-983.

54 Duregon E, Volante M, Cappia S, et al. Oncocytic adrenocortical tumors: diagnostic algorithm and mitochondrial DNA profile in 27 cases. Am J Surg Pathol 2011;35:1882-1893.

55 Duregon E, Volante M, Giorcelli J, et al. Diagnostic and prognostic role of steroidogenic factor 1 in adrenocortical carcinoma: a validation study focusing on clinical and pathologic correlates. Hum Pathol 2013;44:822-828.

56 Bland JM, Altman DG. Measuring agreement in method comparison studies. Stat Methods Med Res 1999;8:135-160.

57 Yamaguchi T, Fujimori T, Tomita S, et al. Clinical validation of the gastrointestinal NET grading system: Ki67 index criteria of the WHO 2010 classification is appropriate to predict metastasis or recurrence. Diagn Pathol 2013;8:65.

58 Giordano TJ. The argument for mitotic rate-based grading for the prognostication of adrenocortical carcinoma. Am J Surg Pathol 2011;35:471-473. 